\title{
Cabazitaxel-loaded human serum albumin nanoparticles as a therapeutic agent against prostate cancer
}

This article was published in the following Dove Press journal:

International Journal of Nanomedicine

26 July 2016

Number of times this article has been viewed

\author{
$\mathrm{Na} \mathrm{Qu}^{\prime}$ \\ Robert J Lee ${ }^{1,2}$ \\ Yating Sun' \\ Guangsheng Cai' \\ Junyang Wang' \\ Mengqiao Wang' \\ Jiahui Lu' \\ Qingfan Meng' \\ Lirong Teng' \\ Di Wang' \\ Lesheng Teng ${ }^{1,3}$
}

'School of Life Sciences, Jilin University, Changchun, People's

Republic of China; ${ }^{2}$ Division of Pharmaceutics, College of Pharmacy,

The Ohio State University, Columbus, $\mathrm{OH}, \mathrm{USA}$; ${ }^{3}$ State Key Laboratory of Long-acting and Targeting Drug Delivery System, Yantai, People's Republic of China
Correspondence: Lesheng Teng;

Di Wang

School of Life Sciences, Jilin University,

No 2699, Qianjin Street, Changchun,

|300 | 2, People's Republic of China

$\mathrm{Tel}+8643185168646$

Email tenglesheng@jlu.edu.cn;

jluwangdi@outlook.com
Abstract: Cabazitaxel-loaded human serum albumin nanoparticles (Cbz-NPs) were synthesized to overcome vehicle-related toxicity of current clinical formulation of the drug based on Tween- 80 (Cbz-Tween). A salting-out method was used for NP synthesis that avoids the use of chlorinated organic solvent and is simpler compared to the methods based on emulsion-solvent evaporation. Cbz-NPs had a narrow particle size distribution, suitable drug loading content (4.9\%), and superior blood biocompatibility based on in vitro hemolysis assay. Blood circulation, tumor uptake, and antitumor activity of Cbz-NPs were assessed in prostatic cancer xenograft-bearing nude mice. Cbz-NPs exhibited prolonged blood circulation and greater accumulation of $\mathrm{Cbz}$ in tumors along with reduced toxicity compared to Cbz-Tween. Moreover, hematoxylin and eosin histopathological staining of organs revealed consistent results. The levels of blood urea nitrogen and serum creatinine in drug-treated mice showed that Cbz-NPs were less toxic than Cbz-Tween to the kidneys. In conclusion, Cbz-NPs provide a promising therapeutic for prostate cancer.

Keywords: cabazitaxel, human serum albumin, nanoparticle, drug delivery, toxicity, prostate cancer

\section{Introduction}

Metastatic castration-resistant prostate cancer (mCRPC) is a molecularly heterogeneous disease. ${ }^{1}$ Cabazitaxel $(\mathrm{Cbz})$ is an active chemotherapy drug for taxane-resistant mCRPC. ${ }^{2} \mathrm{Cbz}$ is a microtubule-stabilizing agent that promotes the polymerization of $\beta$-tubulin. This interferes with mitosis and induces apoptosis. $\mathrm{Cbz}$ shows better antiproliferative activity than docetaxel against chemotherapy-resistant tumor cell lines and better antitumor activity in vivo. ${ }^{3}$ However, the clinical product, Jevtana ${ }^{\circledR}$ (contains $60 \mathrm{mg} \mathrm{Cbz}$ in $1.5 \mathrm{~mL}$ polysorbate 80, Cbz-Tween) manifests severe adverse side effects due to the use of polysorbate 80 (Tween 80 ). ${ }^{4,5}$

Nanoparticles (NPs) are promising as delivery vehicles for drugs. ${ }^{6-8}$ For example, Abraxane $^{\circledR}$, which consists of albumin paclitaxel NPs, has shown advantages over Taxol ${ }^{\circledR} .9$ As the most abundant protein in the blood, human serum albumin (HSA) is an attractive macromolecular carrier for drugs. ${ }^{10} \mathrm{HSA}$-based NPs can improve the solubility of drugs and promote tumor uptake. ${ }^{11}$ HSA-based NPs can extend the half-lives of drugs. ${ }^{12,13}$ Currently, an emulsion-solvent evaporation method with high pressure homogenization technique is commonly utilized for the preparation of HSA NPs. However, this method is complex, and toxic chlorinated organic solvent is required. Therefore, a salting-out method was developed that eliminates the need for chlorinated organic solvent.

In this study, cabazitaxel-loaded HSA nanoparticles (Cbz-NPs) were synthesized by salting out and without the use of a cross-linking agent. The NPs were then evaluated 
for pharmacokinetics, tissue biodistribution, and antitumor efficacy. Hemolysis, blood urea nitrogen (BUN), and serum creatinine (CRE) assays were carried out to assess their biocompatibility and toxicity.

\section{Materials and methods Materials}

Cbz was purchased from Yew Biological Technology Co, Ltd (Wuxi, People's Republic of China). HSA (purity 20\%) was obtained from Octapharma (Vienna, Austria). Acetonitrile and methanol (high performance liquid chromatography [HPLC]grade) were obtained from Sigma-Aldrich Co. (St Louis, MO, USA). Other organic solvents of chromatography grade were purchased from Sinopharm Chemical Reagent Beijing Co, Ltd (Beijing, People's Republic of China).

\section{Animals}

Male nude mice (6 weeks old) and Wistar rats ( $\mathrm{n}=84,300 \pm 10 \mathrm{~g}$ ) were purchased from the Laboratory Animal Center of Jilin University (Changchun, People's Republic of China). They were maintained in a room under 12 hours light/dark cycles with free access to water. All animals were housed at a temperature of $22^{\circ} \mathrm{C} \pm 2{ }^{\circ} \mathrm{C}$. The entire animal protocol prior to conducting the experiments was reviewed and approved by the Institution Animal Ethics Committee (Jilin University) (license No.SCXK-(JI) 2011-0003) and adhered to the Guidelines on Humane Treatment to Lab Animals (published in 2009).

\section{Preparation of Cbz-NPs}

Cbz-NPs were prepared as described previously. ${ }^{14}$ Briefly, $0.65 \mathrm{~mL} 12 \%$ disodium hydrogen phosphate was injected into $1 \mathrm{~mL}$ ethyl alcohol with $10 \mathrm{mg} \mathrm{Cbz}$ with intensive mixing. Then, $0.5 \mathrm{~mL} 20 \% \mathrm{HSA}$ at $65^{\circ} \mathrm{C}$ was injected into this solution with stirring to salt-out $\mathrm{Cbz}$ bound to HSA. Next, the mixture was poured into deionized water at $65^{\circ} \mathrm{C}$ under rapid stirring to induce formation of NPs. After that, the temperature was reduced to $8^{\circ} \mathrm{C}$ rapidly. Ethanol and unencapsulated $\mathrm{Cbz}$ were removed through ultrafiltration. Finally, the sample was freeze-dried without adding a cryoprotectant.

\section{Physicochemical characterization of Cbz-NPs}

The particle size of the NPs resuspended in $0.9 \% \mathrm{NaCl}$ was determined by dynamic light scattering on a Nicomp 380 ZLS from Particle Sizing System (Port Richey, FL, USA). The morphology of Cbz-NPs was evaluated by scanning electron microscopy (JSM-6700F, JEOL, Tokyo, Japan). One drop of properly diluted suspension was deposited onto the surface of a silicon wafer and air-dried. Then the sample was sputtercoated with platinum and imaged by the microscope.

\section{HPLC analysis of Cbz and drug loading content}

Lyophilized NPs were dissolved in acetonitrile. The solutions were sonicated for 15 minutes, centrifuged at 12,000 rpm for 10 minutes, and then $20 \mu \mathrm{L}$ of supernatant from each tube was injected into an HPLC system (LC-20AD, Shimadzu Corporation, Kyoto, Japan) with a HC-C18 column (4.6×150 mm, $5 \mu \mathrm{m}$ particle size, Dikma Technologies, Beijing, People's Republic of China). The mobile phase consisted of water/acetonitrile using a common gradient elute method as described previously. ${ }^{12}$ The flow rate was set to $1.0 \mathrm{~mL} /$ minute; ultraviolet detection wavelength was set to $230 \mathrm{~nm}$; column temperature was maintained at $35^{\circ} \mathrm{C}$.

Loading content (LC) was calculated as follows:

$\mathrm{LC}(\%)=\frac{\text { The amount of } \mathrm{Cbz}}{\text { The weight of lyophilized nanoparticles }} \times 100 \%$

\section{Hemolysis assay}

The hemolytic potentials of Cbz-Tween and Cbz-NPs were evaluated. Cbz-Tween (dissolved in Tween 80 and 13\% [w/w] ethanol in water at ratio of 15:57 [w/w]) and Cbz-NPs (dissolved in $0.9 \%$ saline) at various concentrations were incubated with red blood cells (RBCs) in a suspension at $37^{\circ} \mathrm{C}$ for 2 hours and then centrifuged at 3,000 rpm for 10 minutes. Saline solution and distilled water were used as negative ( $0 \%$ lysis) and positive controls (100\% lysis), respectively. The absorbance of the supernatant was measured at $540 \mathrm{~nm} .{ }^{15}$ The hemolysis ratio was calculated using the following equation:

$$
\text { Hemolysis ratio } \%=\frac{A_{\text {sample }}-A_{\text {negative }}}{A_{\text {positive }}-A_{\text {negative }}} \times 100
$$

where $A_{\text {sample }}, A_{\text {negative }}$, and $A_{\text {positive }}$ represent absorbance of the samples, negative and positive controls, respectively.

\section{Pharmacokinetic study}

Pharmacokinetic analysis was performed using male Wistar rats $(n=12,300 \pm 10 \mathrm{~g})$. The rats were fasted overnight (with free access to water) before the study. Twelve rats were divided into two groups. Heparinized blood samples from 
the ophthalmic vein were collected at $0.25,0.5,0.75,1,2,4$, $6,8,12,24,48$, and 72 hours and immediately centrifuged at $8,000 \mathrm{rpm}$ for 10 minutes to obtain plasma supernatant. The plasma samples were stored at $-20^{\circ} \mathrm{C}$ until HPLC analysis.

\section{Tissue distribution}

PC3 cells $\left(1 \times 10^{6}\right.$ cells/mouse $)$ were injected subcutaneously into male BALB/C nude mice ( 6 weeks old, $n=6$ ). When the average tumor size reached $100 \mathrm{~mm}^{3}, \mathrm{Cbz}$-Tween or Cbz-NPs was injected at a $\mathrm{Cbz}$ dose of $8 \mathrm{mg} / \mathrm{kg}$. At preset time points of $0.5,6$, and 12 hours, the mice were euthanized. Heart, lung, spleen, kidney, liver, and tumor samples were collected. Tissue samples were rinsed with normal saline, weighed, and stored at $-80^{\circ} \mathrm{C}$ until analysis.

The plasma and tissue samples were prepared for analysis using HPLC as described earlier in HPLC analysis of Cbz. Tissues were homogenized with two volumes of saline; $10 \mu \mathrm{L}$ of internal standard solutions $(10 \mu \mathrm{g} / \mathrm{mL}$ diazepam $)$ was added into $50 \mu \mathrm{L}$ plasma or tissue samples with thorough mixing. Then, $50 \mu \mathrm{L}$ of acetonitrile was added. The solution was vortexed for 1 minute and $600 \mu \mathrm{L}$ of chloroform was then added, followed by vortex mixing for 1 minute. After centrifugation at $12,000 \mathrm{rpm}$ for 10 minutes, the supernatant organic solvent was dried at $37^{\circ} \mathrm{C}$ under a mild stream of nitrogen. The residue was resuspended in $50 \mu \mathrm{L}$ of acetonitrile. Finally, $10 \mu \mathrm{L}$ of this solution was injected into HPLC for the determination of $\mathrm{Cbz}$ concentrations.

\section{In vivo antitumor activity assay}

Human prostate cancer PC 3 cells $\left(1 * 10^{6}\right.$ cells/mouse) were injected in the right flank region of male $\mathrm{BALB} / \mathrm{C}$ nude mice ( 6 weeks of age, approximately $20 \mathrm{~g}$ ). Mice were divided into three groups ( $\mathrm{n}=6)$ and treated with Cbz-Tween $(8 \mathrm{mg} / \mathrm{kg}) \mathrm{Cbz}-$ NPs $(8 \mathrm{mg} / \mathrm{kg} \mathrm{Cbz})$ or saline as control, respectively. When the average tumor volume reached $150 \mathrm{~mm}^{3}$, mice were injected from the tail vein every 3 days. Change in the body weight of mice was monitored. Tumor volume was calculated using the formula: tumor volume $=0.5 \times$ major diameter $\times$ minor diameter ${ }^{2}$

After 3 weeks, the mice were sacrificed and dissected to obtain their organs and tumors. Tissue samples were fixed with a formalin solution and embedded in paraffin blocks and sectioned into $10 \mu \mathrm{m}$ thick slices. The slices were stained with hematoxylin and eosin and then imaged under a microscope (Nikon Instruments, Tokyo, Japan).

\section{BUN and serum CRE assays}

To evaluate the changes in kidney function after Cbz-Tween or Cbz-NPs treatment, the levels of BUN and CRE in serum were measured using a kit from NanJing JianCheng Bioengineering Institute (Nanjing, People's Republic of China) according to the manufacturer's instructions. Heparinized blood samples were collected after either $\mathrm{Cbz}$-Tween or Cbz-NPs treatment (saline treatment was used as control) for 24 hours. Then, the supernatant was obtained after centrifugation at 8,000 rpm for 10 minutes and analyzed for Cbz concentration.

\section{Statistical analysis}

Statistical analysis was performed using analysis of variance. Significance cutoff was designated at $P<0.05$.

\section{Results \\ Physicochemical characterization of Cbz-NPs}

A schematic diagram of Cbz-NPs preparation is given in Figure 1A. Lyophilized NPs and NPs redissolved in saline were shown in Figure 1B and Figure 1C, respectively. As demonstrated by the scanning electron microscopy image in Figure 1D, the morphology of NPs is that of a spherical shape with an average size of $110-140 \mathrm{~nm}$. The LC was $4.9 \%$.

\section{Hemolysis assay}

To determine whether the Cbz-NPs formulation is safe for intravenous injection, a hemolysis assay was carried out. As shown in Figure 2, the hemolytic activity was found to be concentration dependent. The hemolytic activity of Cbz-Tween in red blood cells was relatively high and reached $14.93 \%$ at a concentration of $0.02 \mathrm{mg} / \mathrm{mL}$. However, Cbz-NPs exhibited no significant hemolytic activities (below 5.0\%), except at the highest concentration tested $(0.05 \mathrm{mg} / \mathrm{mL}$ of Cbz), suggesting excellent safety for intravenous injection. ${ }^{16}$

\section{Pharmacokinetic study}

The plasma concentration time profiles for $\mathrm{Cbz}$ are shown in Figure 3. Following intravenous administration of CbzTween or Cbz-NPs solution at a dose of $8 \mathrm{mg} / \mathrm{kg}$, at an early time point ( 5 minutes), the plasma $\mathrm{Cbz}$ concentrations showed significant difference: $6.61 \mu \mathrm{g} / \mathrm{mL}$ for Cbz-NPs treatment versus $3.19 \mu \mathrm{g} / \mathrm{mL}$ for Cbz-Tween. Both groups followed similar clearance patterns. However, drug plasma concentration of Cbz-NPs was consistently higher than that of Cbz-Tween at each time point. Therefore, $\mathrm{Cbz}$ in NPs cleared more slowly from the circulation compared to $\mathrm{Cbz}$ in Tween. At 48 hours postadministration, the plasma drug concentration of Cbz-NPs was $140 \mathrm{ng} / \mathrm{mL}$, while the Cbz was barely detected in the group treated with Cbz-Tween. 


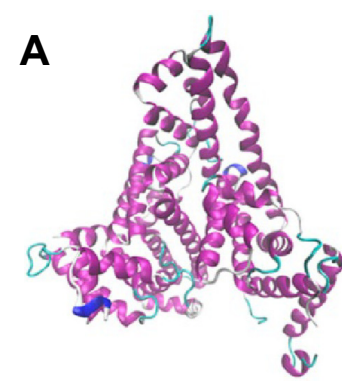

HSA

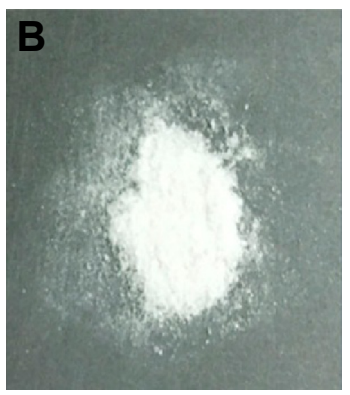

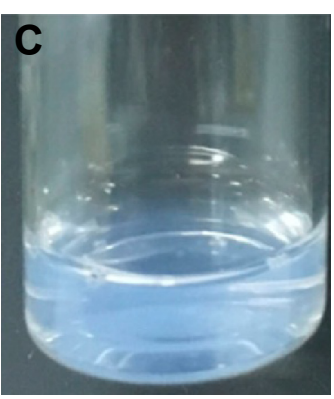

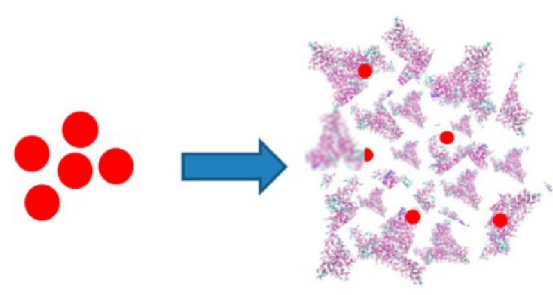

$\mathrm{Cbz}$

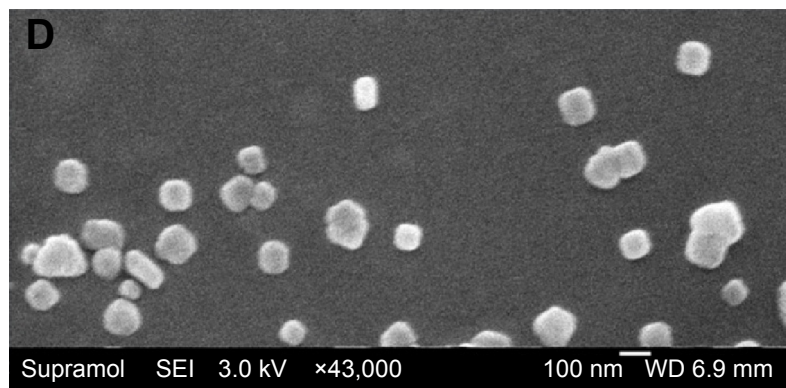

Figure I Physicochemical characterization of Cbz-NPs.

Notes: (A) A schematic diagram of Cbz-NPs preparation. (B) Lyophilized NPs. (C) NPs redissolved in saline. (D) SEM image of Cbz-NPs.

Abbreviations: Cbz-NPs, cabazitaxel-loaded human serum albumin nanoparticles; HSA, human serum albumin; SEM, scanning electron microscopy.
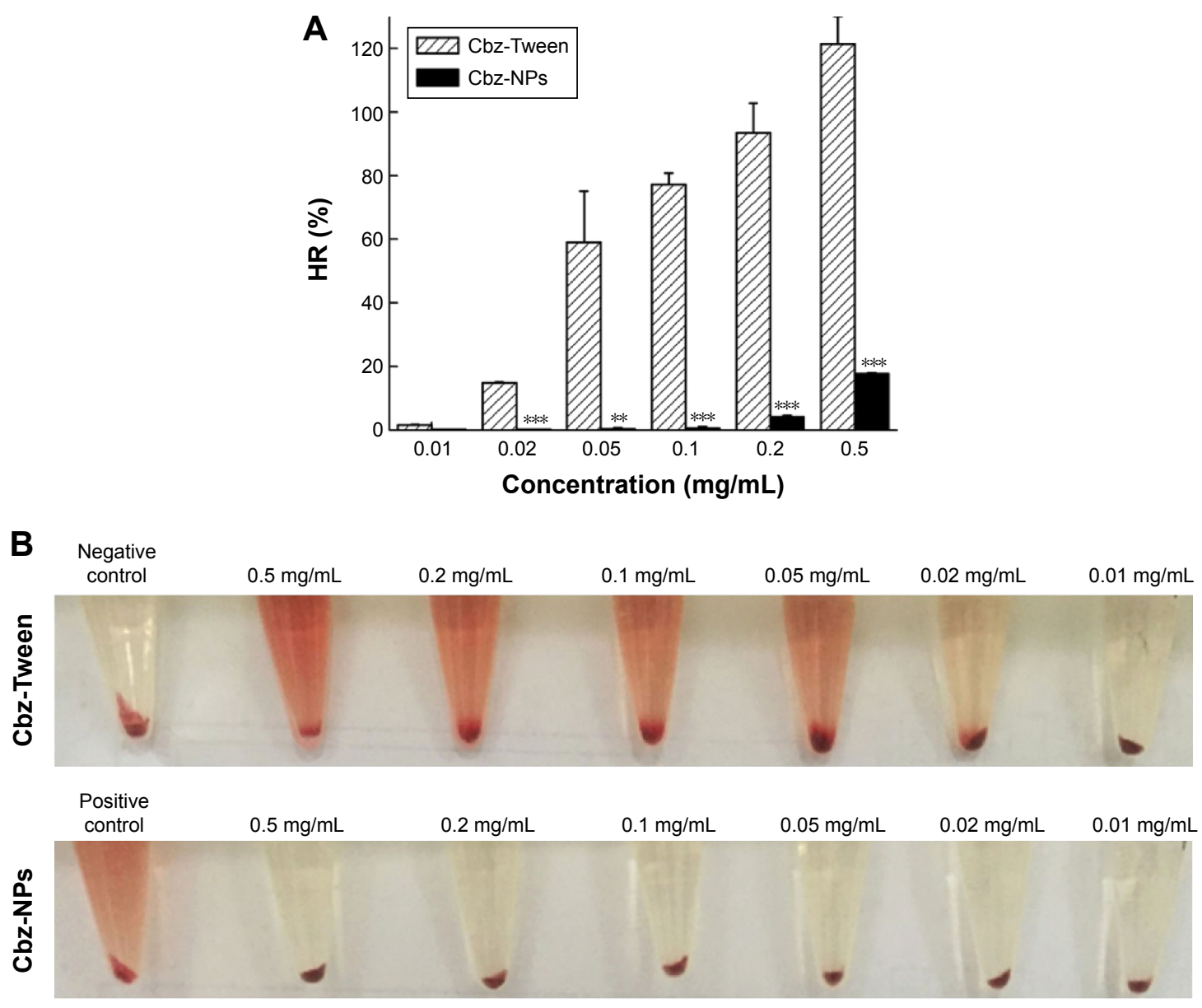

Figure 2 In vitro hemolysis assay of Cbz-Tween and Cbz-NPs.

Notes: $(\mathbf{A}) \mathrm{HR}(\%)$ of various concentration of Cbz. (B) Images of RBCs treated with Cbz-Tween or Cbz-NPs. Data are expressed as mean $\pm \mathrm{SE}$ ( $\mathrm{n}=3$ ). $* * P<0.0 \mathrm{I}$; $* * * P<0.001$.

Abbreviations: Cbz-NPs, cabazitaxel-loaded human serum albumin nanoparticles; HR (\%), hemolysis ratio; RBC, red blood cells; SE, standard error. 
A

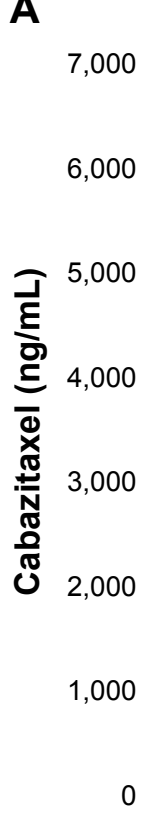

B

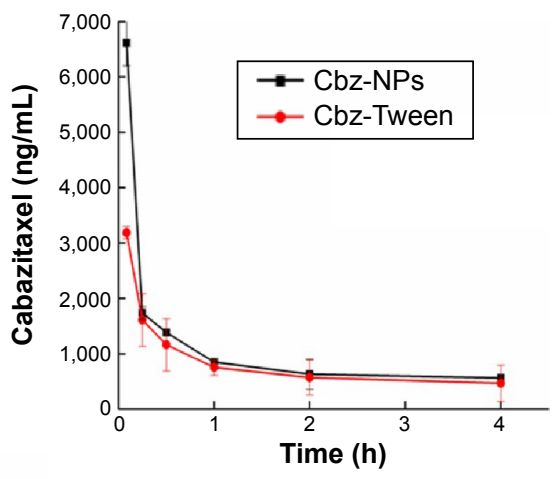

Figure 3 The mean plasma concentration-time profiles for Cbz.

Notes: (A) The concentration-time curve of Cbz in rat plasma after Cbz-NPs and Cbz-Tween administration. (B) The subtle differences at early time points are shown as an inset.

Abbreviations: Cbz-NPs, cabazitaxel-loaded human serum albumin nanoparticles; h, hours.

The pharmacokinetic parameters obtained are presented in Table 1. The maximum plasma concentration $\left(C_{\max }\right)$, the time of maximal concentration $\left(T_{\max }\right)$, the half-life $\left(T_{1 / 2}\right)$, the area under the curve $\left(A U C_{0-\infty}\right)$, and mean residence time $\left(M R T_{0-\infty}\right)$ were calculated. $T_{\max }$ values of the two formulations were the same at the first detection point (5 minutes). Compared to that of Cbz-Tween treatment, the half-life value of NPs was higher, indicating a longer circulation time. Moreover, the $A U C_{0-\infty}$ and $M R T_{0-\infty}$ values of $\mathrm{Cbz}$ following NPs treatment were significantly higher than those following Cbz-Tween treatment.

\section{Tissue distribution}

The tissue concentrations of $\mathrm{Cbz}$ at $0.5,6$, and 12 hours after intravenous administration at a dose of $8 \mathrm{mg} / \mathrm{kg}$ are shown in Figure 4. The distribution of two formulations was determined in major tissues, including heart, liver, spleen, lung, kidney, and tumor.
At the early time point of 0.5 hour, the drug concentrations in most organs were higher following Cbz-NPs treatment than Cbz-Tween. The concentration in the heart was significantly lower following Cbz-NPs treatment at every time point. The liver accumulation of Cbz after Cbz-NP treatment was considerably greater than that after Cbz-Tween treatment at all time points. However, at the early point time, the $\mathrm{Cbz}$ level in the tumor of mice receiving NPs was lower than that after Cbz-Tween therapy. However, the tumor drug level was much higher for the Cbz-NP treatment group 12 hours post-injection.

\section{In vivo antitumor activity assay}

We evaluated the therapeutic efficacy of Cbz-NPs and CbzTween in a murine prostate cancer xenograft model. The average tumor volume and animal weight were monitored during the treatment period (Figure 5A and B). Both Cbz-NPs and Cbz-Tween suppressed the growth of tumors. On day

Table I Plasma pharmacokinetic parameters

\begin{tabular}{llllll}
\hline Formulation & $\boldsymbol{C}_{\max }(\mathrm{ng} / \mathrm{mL})$ & $\boldsymbol{T}_{\max }(\mathrm{h})$ & $\boldsymbol{T}_{1 / 2}(\mathbf{h})$ & AUC $_{0-\infty}$ & $\mathbf{M R T}_{0-\infty}$ \\
\hline Cbz-Tween & $3,190 \pm 389$ & $0.083 \pm 0.000$ & $10.9 \pm 2.71$ & $11,675 \pm 1,219$ & $13.0 \pm 2.31$ \\
Cbz-NPs & $6,615 \pm 612^{*}$ & $0.083 \pm 0.000$ & $34.1 \pm 3.33$ & $1,5501 \pm 2,172$ & $16.1 \pm 2.59$ \\
\hline
\end{tabular}

Notes: Data are expressed as mean \pm SE $(n=6)$. $* P<0.05$.

Abbreviations: $A \cup C_{0-\infty}$, area under the curve; $C_{\text {max }}$ maximum plasma concentration; Cbz-NP, cabazitaxel-loaded human serum albumin nanoparticles; $h$, hours; MRT, mean residence time; $\mathrm{SE}$, standard error; $T_{\text {max }}$, time of maximal concentration; $T_{1 / 2}$, half-life. 

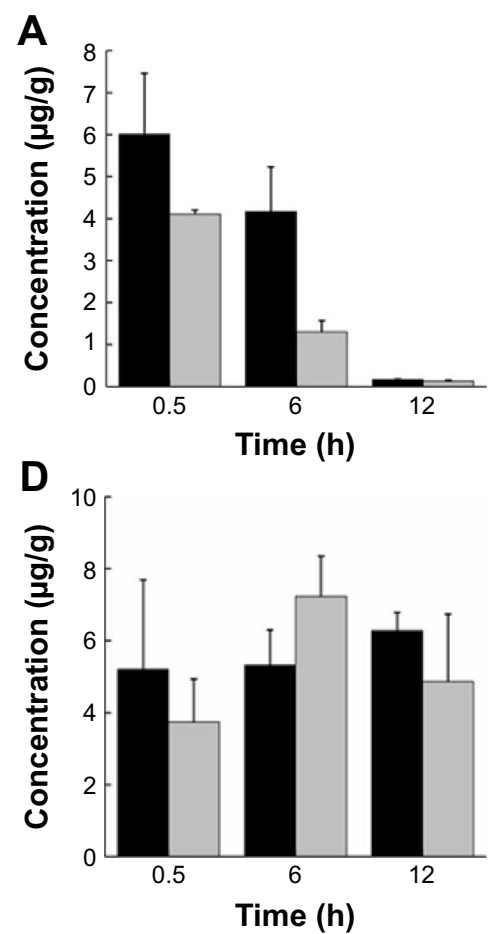

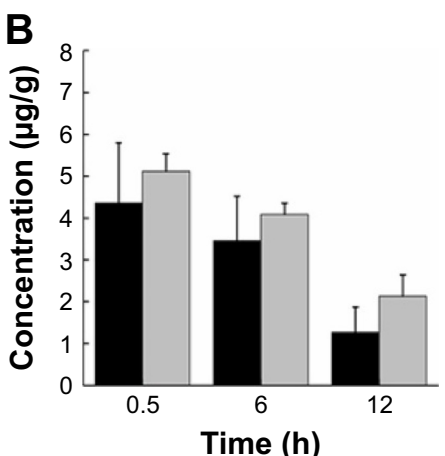

E

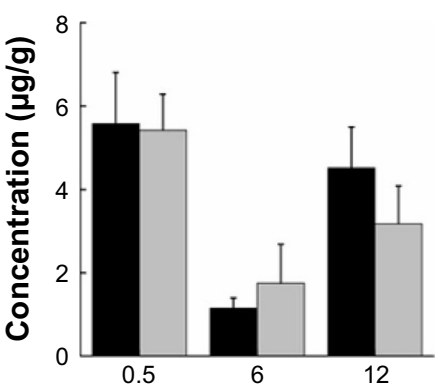

Time (h)

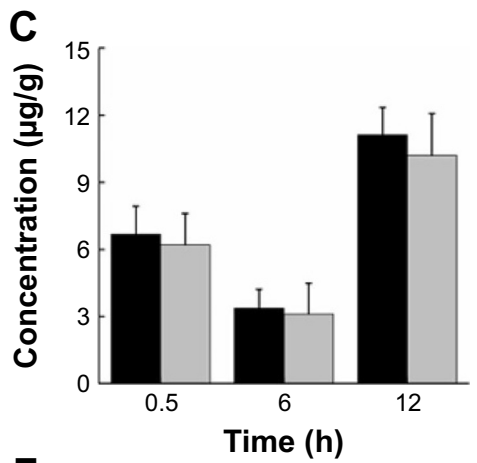

$\mathbf{F}$

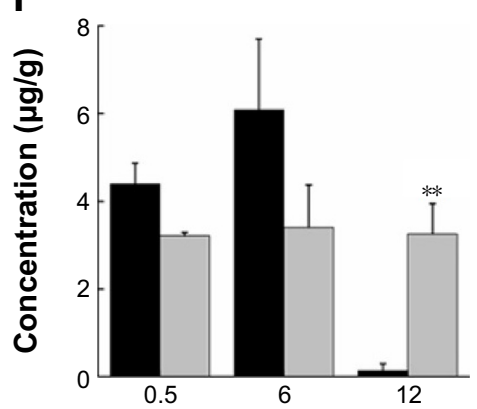

Time (h)

Figure 4 Tissue distribution of $\mathrm{Cbz}$ to the organs after treatments with either Cbz-NPs or Cbz-Tween.

Notes: (A) Heart, (B) liver, (C) spleen, (D) lung, (E) kidney, and (F) tumor. Data are expressed as mean $\pm S E(n=6)$. $* * P<0.0$ I.

Abbreviations: Cbz-NPs, cabazitaxel-loaded human serum albumin nanoparticles; h, hours; SE, standard error.

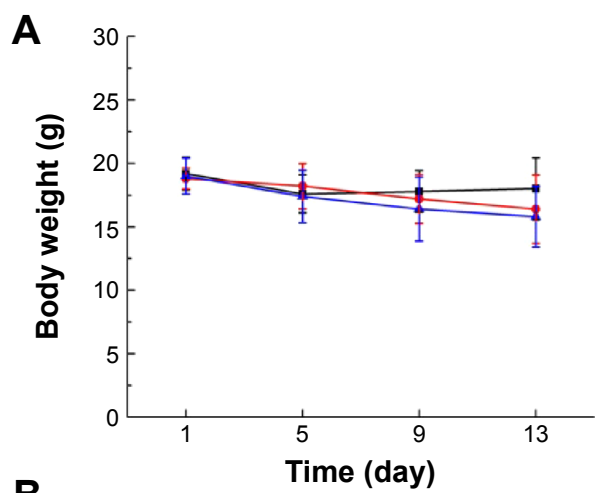

B

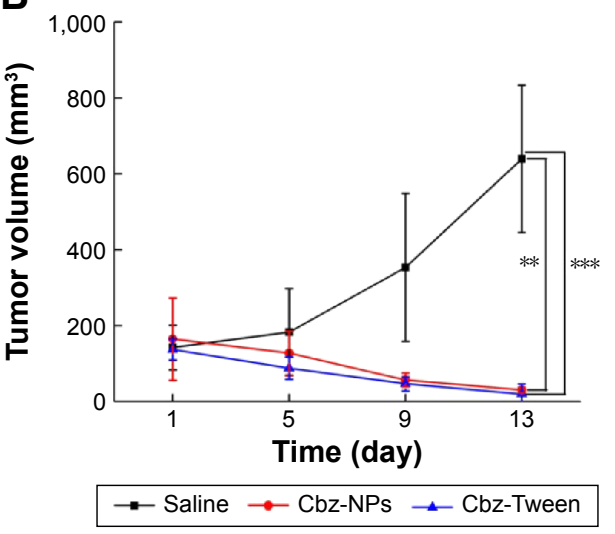

C

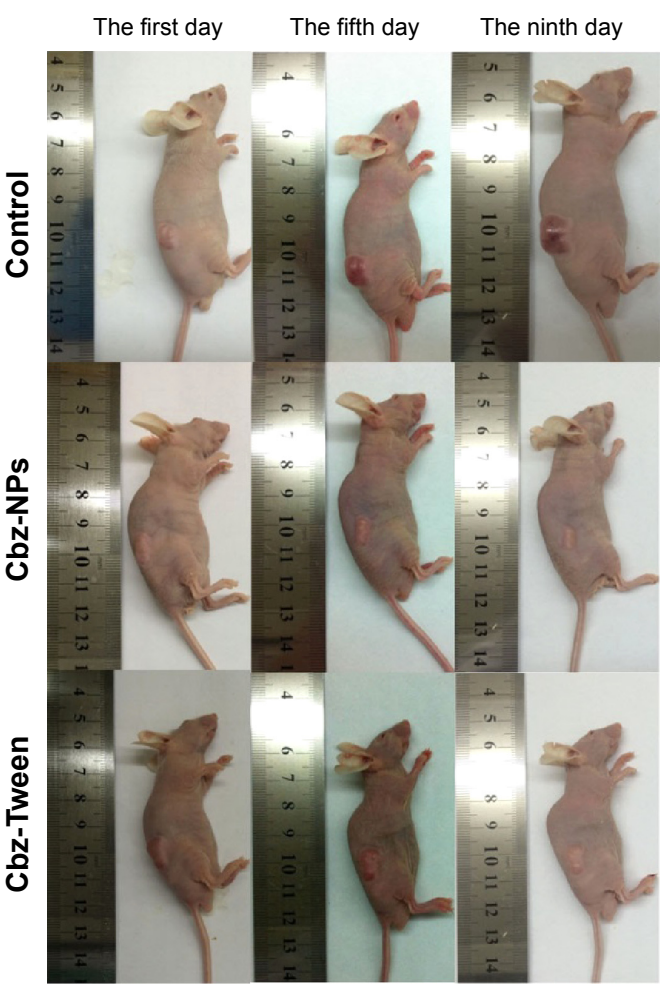

Figure 5 Antitumor activity of Cbz-NPs treatment on prostate cancer tumor-bearing mice compared to Cbz-Tween treatment.

Notes: (A) Body weight of the mice during the experiment. (B) Antitumor effect of Cbz-NPs and Cbz-Tween tumor-bearing mice. (C) Images of the nude mice after intravenous injections of Cbz-Tween and Cbz-NPs, saline was used as a control. Data are expressed as mean $\pm S E(n=7)$. $* * P<0.0 \mathrm{I} ; * * * P<0.00 \mathrm{I}$.

Abbreviations: Cbz-NPs, cabazitaxel-loaded human serum albumin nanoparticles; SE, standard error. 

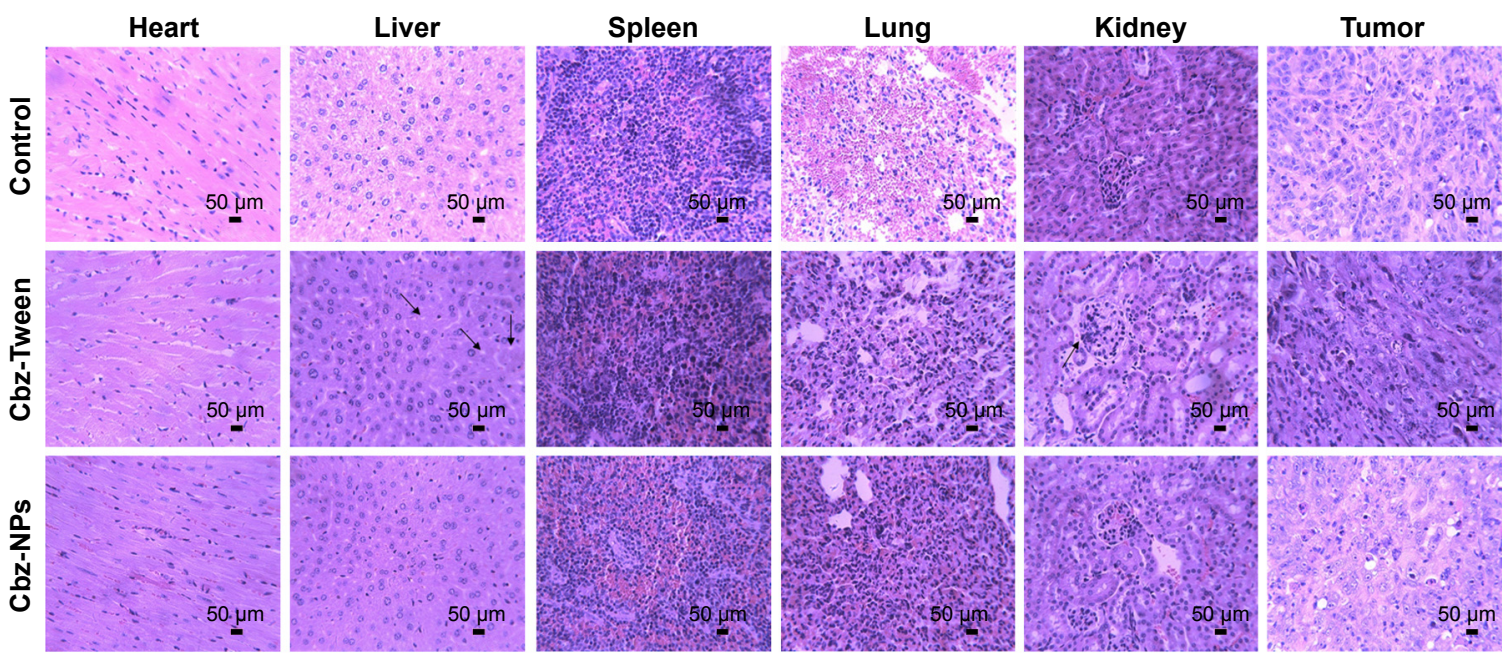

Figure 6 The pathological hematoxylin and eosin staining analysis of tissue sections. Note: Arrows were used to indicate relevant areas of fat liver and spleen infiltration. Abbreviation: Cbz-NPs, cabazitaxel-loaded human serum albumin nanoparticles.

13, the tumor sizes were similar for all treatment groups and the tumors almost disappeared (below $30 \mathrm{~mm}^{3}$ ). Due to the side effect of Cbz-Tween and the tumor development, both treatment groups exhibited a reduction in body weight after drug therapy. Mice treated with Cbz-Tween showed slightly greater reduction in body weight compared to those treated with Cbz-NPs. However, the difference was not statistically significant. The changes of tumor volume were observed visually in Figure 5C.

Then, the tumor tissues were further analyzed by histological staining. As shown in Figure 6, there was extensive tumor cell death. However, the structure in Cbz-NPs group is much looser compared to the Tween group. The liver, spleen, and kidney of Cbz-Tween-treated mice displayed histological changes in sharp contrast to saline and Cbz-NPs treatments groups. Fat liver and spleen infiltration were found in the group of Cbz-Tween treatment (Figure 6). Glomerular atrophy was also apparent in this treatment group, which has been indicated with arrows in relevant areas in Figure 6. However, histological changes in Cbz-NPs were not obvious.

\section{The levels of BUN and serum CRE}

The levels of BUN and CRE in serum are routinely used to assess kidney function. As shown in Table 2, the level of BUN

Table 2 The levels of BUN and serum CRE (mean \pm SD, $n=6$ )

\begin{tabular}{llll}
\hline Subjects number & Control & Cbz-NPs & Cbz-Tween \\
\hline BUN (mg/dL) & $26.0102 \pm 0.242$ & $37.8765 \pm 6.831$ & $57.9382 \pm I I .404^{* *}$ \\
CRE (mg/dL) & $0.3520 \pm 0.135$ & $0.2343 \pm 0.030$ & $0.7270 \pm 0.462$ \\
\hline
\end{tabular}

Notes: Data are expressed as mean $\pm S D(n=6)$. $* * p<0.01$.

Abbreviations: BUN, blood urea nitrogen; Cbz-NPs, cabazitaxel-loaded human serum albumin nanoparticles; CRE, creatinine; SD, standard deviation. for Cbz-Tween treatment increased significantly $(P<0.01)$ compared to untreated control and the level of CRE in serum was $0.72 \mathrm{mg} / \mathrm{dL}$, which was outside of the normal range: $0.1-0.6 \mathrm{mg} / \mathrm{dL}$; while, these parameters for mice receiving Cbz-NPs treatment were similar to those of the control.

\section{Discussion}

Globally, $>900,000$ new cases of mCRPC are diagnosed each year, resulting in over 250,000 deaths. ${ }^{17} \mathrm{Cbz}$, a novel tubulin-binding taxane, is an effective anticancer drug for mCRPC treatment and its clinical application is increasing. ${ }^{18}$ However, the toxicity of commercial Cbz-Tween is significant due to the use of polysorbate 80 , suggesting a need for the development of an improved formulation.

It is well-known that albumin-based NPs can be used for delivery of drugs with low solubility. HSA can bind hydrophobic drugs and has excellent biocompatibility. We prepared Cbz-NPs without using cross-linking agents and using a method based on salting-out. Previously, we had reported about the in vitro properties of these NPs. ${ }^{14}$ To investigate the therapeutic potential of Cbz-NPs, in vivo studies are conducted and reported in this article.

The NPs prepared as described in the "Materials and methods" section had a narrow particle size distribution, high LC, and extended in vivo circulation time. It is crucial to establish the blood compatibility of the drug carriers designed for intravenous injection. Therefore, evaluation of the hemolytic activity in vitro is necessary. Figure 2 shows excellent blood biocompatibility of Cbz-NPs, suggesting suitability for intravenous administration.

The pharmacokinetics of Cbz-NPs was studied using Wistar rats with Cbz-Tween solution as a control. The 
$C_{\max }$ obtained with Cbz-NPs was much higher than that of Cbz-Tween at the first time point. Considering the abundance of endogenous albumin in blood, it might be inevitable that the lipophilic Cbz transfers from NPs and associates with endogenous albumin while in circulation. ${ }^{19}$ Collectively, NP delivery can diminish clearance and facilitate long-circulating $\mathrm{Cbz}$ in blood, resulting in increases in $A U C_{0-\infty}, M R T_{0-\infty}$, and $T_{1 / 2}$.

In tissue distribution study, Cbz-NPs reduced the accumulation of drug in heart, spleen, and kidney, which reduced toxicity in these organs. Increased delivery to the tumor tissue was obtained with the Cbz-NPs. This might be attributed to passive targeting by enhanced permeability and retention effect ${ }^{20}$ and preferential tumor uptake due to elevated caveolin-1 protein and secreted protein acidic and rich in cysteine in the tumor.

Antitumor activity of Cbz-NPs in vivo was evaluated by the tumor growth inhibition on prostatic cancer tumor xenograft-bearing nude mice. The inhibitory effect of Cbz-NPs is almost equal to that of Cbz-Tween, which indicates similar levels of antitumor activity.

Pathological hematoxylin and eosin staining examinations of major organs following Cbz-NPs treatment showed reduced toxicity relative to $\mathrm{Cbz}-\mathrm{Tw}$ een. Potential toxic reactions were noticed in the liver, spleen, and kidney of Cbz-Tween treatment. Moreover, Cbz-Tween treatment also adversely affected kidney function. The structure in CbzNPs group is much looser compared to the Tween group, indicating a potentially targeted therapeutic effect in the tumor tissue for the NP group. CRE in serum has been the most frequently employed marker to estimate glomerular filtration rate. Plasma CRE increase has been linked to the progression of kidney disease. ${ }^{21,22}$ The accumulation of CRE following Cbz-Tween treatment due to the slow CRE clearance may be attributed to treatment-related kidney failure. The significant rise in BUN level in the Cbz-Tween treatment group ( $P<0.01$, compared to control) is probably due to low volume of distribution of urea, which was caused by impaired kidney function. ${ }^{23}$ Meanwhile, the levels of CRE and BUN in serum for Cbz-NPs-treated mice showed only slight changes, suggesting reduced renal toxicity.

Based on these results, delivery of Cbz using albuminbased NPs has resulted in a reduction in toxic manifestation of drug, indicating promising potential of this platform of drug delivery.

\section{Conclusion}

The results of this study showed that Cbz-NPs have significant safety advantages compared to Cbz-Tween. Superior blood biocompatibility was demonstrated in hemolysis assay in vitro. Cbz-NPs showed prolonged blood circulation and enhanced accumulation of $\mathrm{Cbz}$ in tumors along with reduced toxicity. Therefore, Cbz-NPs have potential in future clinical therapy of prostatic cancer.

\section{Disclosure}

The authors report no conflicts of interest in this work.

\section{References}

1. Mellado B, Jimenez N, Marin-Aguilera M, Reig O. Diving into cabazitaxel mode of action; more than a taxane for the treatment of castration resistance prostate cancer patients. Clin Genitourin Cancer. Epub 2015 Dec 28.

2. Zhuang B, Du L, Xu H, et al. Self-assembled micelle loading cabazitaxel for therapy of lung cancer. Int J Pharm. 2016;499(1-2): $146-155$.

3. Moriceau G, Guillot A, Pacaut C, et al. Translating clinical evidencebased medicine into the real world: single-center experience with cabazitaxel in metastatic prostate cancer patients. Chemotherapy. 2016; 61(3):127-133.

4. He Z, Schulz A, Wan X, et al. Poly(2-oxazoline) based micelles with high capacity for 3rd generation taxoids: preparation, in vitro and in vivo evaluation. J Control Release. 2015;208:67-75.

5. Coors EA, Seybold H, Merk HF, Mahler V. Polysorbate 80 in medical products and nonimmunologic anaphylactoid reactions. Ann Allergy Asthma Immunol. 2005;95(6):593-599.

6. Gao Y, Xie J, Chen H, et al. Nanotechnology-based intelligent drug design for cancer metastasis treatment. Biotechnol Adv. 2014;32(4): 761-777.

7. Kraft JC, Freeling JP, Wang Z, Ho RJ. Emerging research and clinical development trends of liposome and lipid nanoparticle drug delivery systems. J Pharm Sci. 2014;103(1):29-52.

8. Goyal R, Macri LK, Kaplan HM, Kohn J. Nanoparticles and nanofibers for topical drug delivery. J Control Release. Epub 2015 Oct 28.

9. Reddy LH, Bazile D. Drug delivery design for intravenous route with integrated physicochemistry, pharmacokinetics and pharmacodynamics: illustration with the case of taxane therapeutics. Adv Drug Deliv Rev. 2014;71:34-57.

10. Cui W, Wang A, Zhao J, Yang X, Cai P, Li J. Layer by layer assembly of albumin nanoparticles with selective recognition of tumor necrosis factor-related apoptosis-inducing ligand (TRAIL). J Colloid Interface Sci. 2016;465:11-17

11. Wan X, Zheng X, Pang X, Zhang Z, Zhang Q. Incorporation of lapatinib into human serum albumin nanoparticles with enhanced anti-tumor effects in HER2-positive breast cancer. Colloids Surf B Biointerfaces. 2015;136:817-827.

12. Qu G, Wu X, Yin L, Zhang C. N-octyl-O-sulfate chitosan-modified liposomes for delivery of docetaxel: preparation, characterization, and pharmacokinetics. Biomed Pharmacother. 2012;66(1):46-51.

13. Weiszhar Z, Czucz J, Revesz C, Rosivall L, Szebeni J, Rozsnyay Z. Complement activation by polyethoxylated pharmaceutical surfactants: Cremophor-EL, Tween-80 and Tween-20. Eur J Pharm Sci. 2012;45(4): 492-498.

14. Qu N, Sun YT, Xie J, Teng LS. Preparation and evaluation of selfassembling HSA nanoparticles for cabazitaxel in vitro. Anti-Cancer Agents Med Chem. Epub 2016 May 25.

15. Zhang J, Han J, Zhang XL, et al. Polymeric nanoparticles based on chitooligosaccharide as drug carriers for co-delivery of all-trans-retinoic acid and paclitaxel. Carbohydr Polym. 2015;129:25-34.

16. Cao J, Chen YW, Wang X, Luo XL. Enhancing blood compatibility of biodegradable polymers by introducing sulfobetaine. J Biomed Mater Res A. 2011;97(4):472-479. 
17. Singer EA, Srinivasan R. Intravenous therapies for castration-resistant prostate cancer: toxicities and adverse events. Urol Oncol. 2012; 30(4 Suppl):S15-S19.

18. Karavelioglu E, Gonul Y, Aksit H, et al. Cabazitaxel causes a dosedependent central nervous system toxicity in rats. J Neurol Sci. 2016; 360:66-71.

19. Li C, Li Y, Gao Y, et al. Direct comparison of two albumin-based paclitaxel-loaded nanoparticle formulations: is the crosslinked version more advantageous? Int J Pharm. 2014;468(1-2):15-25.

20. Pourjavadi A, Tehrani ZM. Mesoporous silica nanoparticles with bilayer coating of poly(acrylic acid-co-itaconic acid) and human serum albumin (HSA): A pH-sensitive carrier for gemcitabine delivery. Mater Sci Eng C Mater Biol Appl. 2016;61:782-790.
21. Ognibene A, Grandi G, Lorubbio M, et al. KDIGO 2012 clinical practice guideline CKD classification rules out creatinine clearance 24 hour urine collection? Clin Biochem. 2016;49(1):85-89.

22. Shashidhar KN, Munilakshmi U, Prabhavathi K, Reddy M, Lakshmaiah V. Potential role of uric acid in correlation with epidemics of hypertension and albumin creatinine ratio in diabetic nephropathy. Apollo Med. 2015;13(1):24-30.

23. Shavit L, Lifschitz M, Galperin I. Influence of enteric nutrition on blood urea nitrogen (BUN) in very old patients with chronic kidney disease (CKD). Arch Gerontol Geriatr. 2012;54(1):228-231.
International Journal of Nanomedicine

\section{Publish your work in this journal}

The International Journal of Nanomedicine is an international, peerreviewed journal focusing on the application of nanotechnology in diagnostics, therapeutics, and drug delivery systems throughout the biomedical field. This journal is indexed on PubMed Central, MedLine, CAS, SciSearch $®$, Current Contents $\AA /$ Clinical Medicine,

\section{Dovepress}

Journal Citation Reports/Science Edition, EMBase, Scopus and the Elsevier Bibliographic databases. The manuscript management system is completely online and includes a very quick and fair peer-review system, which is all easy to use. Visit http://www.dovepress.com/ testimonials.php to read real quotes from published authors.

Submit your manuscript here: http://www.dovepress.com/international-journal-of-nanomedicine-journal 